\title{
A weapon of endothelial cells for fighting vascular disease
}

\author{
Naruya Tomita and Norio Komai \\ Hypertension Research (2010) 33, 112-113; doi:10.1038/hr.2009.216; published online 8 January 2010
}

$\mathrm{V}$ ascular endothelial cells, of which there are roughly $10^{14}$ in the human body, produce various factors that have important roles in the regulation of vascular tone. An amazing weapon of endothelial cells for fighting vascular disease is endothelial nitric oxide synthase (eNOS), which is an enzyme that generates a key vasoprotective factor, nitric oxide (NO). NO regulates vascular tone by inhibition of platelet aggregation and adhesion, suppression of vascular smooth muscle cell proliferation, prevention of oxidative modification and decrease of proinflammatory gene expression. ${ }^{1}$ All of these factors contribute to the progression of atherosclerosis. Indeed, impaired endothelial function has been found in the arteries of the forearm, coronary arteries and renal vasculature in hypertension, diabetes mellitus, dyslipidemia and coronary artery disease. ${ }^{2}$ Endothelial dysfunction is an early feature of atherosclerosis and vascular disease in humans. ${ }^{3}$ One possible mechanism of endothelial dysfunction is a decrease in NO bioavailability. Endothelial function has been shown to be associated with an imbalance between $\mathrm{NO}$ and ROS, so-called oxidative stress.

In this issue of Hypertension Research, Satoh et al. ${ }^{4}$ focus on endothelial dysfunction and oxidative stress in Dahl salt-sensitive hypertensive rats. The authors have recently developed a novel method to directly detect $\mathrm{NO}$ and ROS at the same time using fluorescent probes. In this paper, the authors show that in the kidney, NO but not ROS was detected in the glomeruli and arterioles of

Division of Nephrology, Department of Internal Medicine, Kawasaki Medical School, Kurashiki, Japan

Dr N Tomita is at the Division of Nephrology, Department of Internal Medicine, Kawasaki Medical School, 577 Matsushima, Kurashiki 701-0192, Japan.

E-mail: tomita@med.kawasaki-m.ac.jp normotensive rats; however, ROS production was increased and NO production was decreased in Dahl salt-sensitive hypertensive rats. The authors also previously reported the same pattern, an imbalance of ROS/NO, indicating increased oxidative stress in the kidneys of diabetic and metabolic syndrome model rats. 5 , What is the cause of the increased oxidative stress? The authors have clearly shown that $\mathrm{NAD}(\mathrm{P}) \mathrm{H}$ oxidase and uncoupled eNOS are major sources of increased oxidative stress in Dahl salt-sensitive hypertensive rat kidneys. An especially noteworthy result is that eNOS uncoupling exists in the glomeruli of Dahl salt-sensitive hypertensive rats. Previously, the authors had reported the existence of uncoupled eNOS in a diabetic model rat kidney. ${ }^{5,7}$ eNOS produces ROS rather than NO under conditions of eNOS uncoupling through a deficiency of tetrahydrobiopterin $\left(\mathrm{BH}_{4}\right)$, an essential cofactor for eNOS (Figure 1). In turn, a deficiency of $\mathrm{BH}_{4}$ caused by ROS leads to downregulation of eNOS. ${ }^{8}$ Supplementation with $\mathrm{BH}_{4}$ improved endothelial function in both

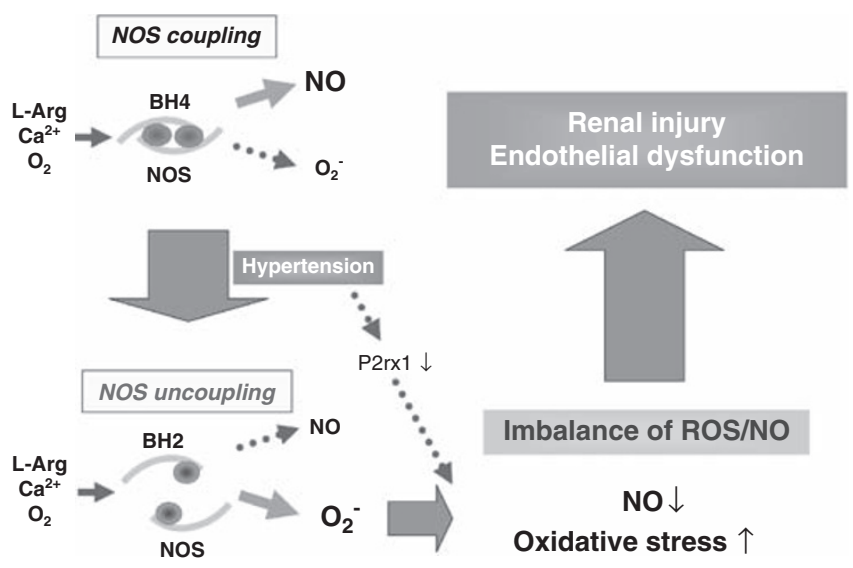

Figure 1 eNOS uncoupling can progress the pathogenesis of endothelial dysfunction. in vitro and in vivo experiments. Moreover, this supplementation effect has already been proven in smokers and patients with hypertension, hypercholesterolemia or chronic heart failure. ${ }^{9-11}$ In this study, the authors examined the expression of GTPCH1, which is the rate-limiting enzyme in $\mathrm{BH}_{4}$ synthesis, and the findings are consistent with those in previous reports. Recently, Higashi et al. ${ }^{12}$ also showed that the grade of oxidative stress correlates with $\mathrm{BH}_{4}$ deficiency and that supplementation with $\mathrm{BH}_{4}$ augmented endothelium-dependent vasodilation in the brachial arteries of elderly patients. Satoh et al. ${ }^{4}$ showed this indirectly by showing decreased plasma levels of $\mathrm{BH}_{4}$ and increased plasma levels of $\mathrm{BH}_{2}$. On the basis of previous findings combined with the data from the current study, it is suggested that $\mathrm{BH}_{4}$ deficiency and decreased eNOS activity cause endothelial dysfunction through an increase in oxidative stress in atherosclerotic patients with hypertension, diabetes and metabolic syndrome. It is true that there are several pathways to increase oxidative stress other than eNOS 
uncoupling; however, in terms of NO bioavailability, eNOS has the most important role to fight vascular disease.

Moreover, the authors have shown an interesting finding that the renal autoregulatory mechanism was impaired in the early stages of hypertension onset. According to the results of this study, the capacity to autoregulate the steady-state renal flow in response to step changes was decreased in Dahl saltsensitive rats fed a high-salt diet compared with those fed a low-salt diet. Then, the authors focused on the P2rxl gene, which encodes a protein belonging to the family of purinoceptors for ATP. This receptor functions as a ligand-gated ion channel with relatively high calcium permeability. Binding to ATP mediates synaptic transmission between neurons and from neurons to smooth muscle; this binding is responsible, for example, for renal microcirculation and for sympathetic vasoconstriction in small arteries, arterioles and vas deferens. ${ }^{13}$ The authors observed decreased mRNA expression of P2rxl in hypertensive rat glomeruli by real-time PCR. Thus, the authors speculate that P2rx1 expression was decreased as a result of renal damage caused by hypertension and that this decrease may have lead to the impairment of renal autoregulation.

In cultured endothelial cells, the application of antioxidants increases eNOS activity via regeneration of $\mathrm{BH}_{4} \cdot{ }^{14}$ Long-term in vivo vitamin $\mathrm{C}$ treatment restored endothelial function and eNOS activity in the aorta of Apoe $^{-1-}$ mice. In patients with coronary heart disease, acute infusion of high-dose vitamin $\mathrm{C}$ has been found to improve endothelial function. Thus, vitamin $\mathrm{C}$ may improve endothelial function; however, a clear mechanism is not yet known. ${ }^{15}$ In addition, there is no evidence showing that vitamin $\mathrm{C}$ supplementation reduces the risk of cardiovascular morbidity or mortality. ${ }^{16}$ Thus, the efficacy of vitamin $\mathrm{C}$ in preventing endothelial dysfunction seems to be controversial. Of the existing drug classes used to treat vascular diseases, angiotensin-converting enzyme inhibitors, angiotensin receptor blockers (ARBs) and statins, have been shown to reduce vascular oxidative stress, improve endothelial dysfunction and ultimately provide a prognostic benefit. Importantly, the authors showed that treatment with an ARB, telmisartan, restored the ROS/NO balance in the kidney and probably in the vasculature, leading to improvement in endothelial dysfunction in the aorta. Moreover, telmisartan has been shown to restore the renal P2rx1 expression to control levels, leading to improvement in renal flow autoregulation. It is well known that ARBs decrease ROS production and that this decrease may have a renoprotective effect. Through suppression of ROS, the authors have elucidated the role of P2rxl in hypertensive renal damage. ARB may regulate renal P2rxl expression and autoregulation in hypertension. The results of this study may strengthen the case for ARB use for renoprotection in hypertensive patients.

\section{CONFLICT OF INTEREST}

The authors declare no conflict of interest.

1 Försterman U, Münzel T. Endothelial nitoric oxide synthase in vascular disease: from marvel to menace. Circulation 2006; 113: 1708-1714.

2 Higashi Y, Noma K, Yoshizumi M, Kihara Y. Endothelial function and oxidative stress in cardiovascular disease. Circ J 2009; 73: 411-418.

3 Ross R. Atherosclerosis: an inflammatory disease. N Engl J Med 1999; 340: 115-126.

4 Satoh M, Haruna Y, Fujimoto S, Sasaki T, Kashihara N. Telmisartan improves endothelial dysfunction and renal autoregulation in Dahl salt-sensitive rats. Hypertens Res (e-pub ahead of print 20 November 2009; doi:10.1038/hr.2009.190).
5 Satoh M, Fujimoto S, Haruna Y, Arakawa S, Horike $\mathrm{H}$, Komai N, Sasaki T, Tsujioka K, Makino H, Kashihara N. $\mathrm{NAD}(\mathrm{P}) \mathrm{H}$ oxidase and uncoupled nitric synthase are major sources of glomerular superoxide in rats with experimental diabetic nephropathy. $A m$ J Physiol Renal Physiol 2005; 288: F1144-F1152.

6 Namikoshi T, Tomita N, Satoh M, Haruna Y, Kobayashi S, Komai N, Sasaki T, Kashihara N. Olmesartan ameliorates renovascular injury and oxidative stress in zucker obese rats enhanced by dietary protein. Am J Hypertens 2007; 20: 1085-1091.

7 Satoh M, Fujimoto S, Haruna Y, Arakawa S, Yada T, Namikoshi T, Haruna Y, Horike H, Sasaki T, Kashihara N. Angiotensin II type 1 receptor blocker ameliorates uncoupled endothelial nitoric oxide synthase in rats with experimental diabetic nephropathy. Nephrol Dial Transplant 2008; 23: 3806-3813.

8 Kuzkaya N, Weissmann N, Harrison DG, Dikalov S. Interactions of peroxynitrate, tetrahydrobiopterin, ascorbic acid, and thiols: implications for uncoupling endothelial nitoric-oxide synthase. J Biol Chem 2003; 278: 22546-22554.

9 Heitzer T, Brockhoff C, Mayer B, Warnholtz A, Mollnau $H$, Simone $H$, Meinertz T, Münzel T. Tetrahydrobiopterin improves endothelium-dependent vasodilation in chronic smokers: evidence for a dysfunctional nitric oxide synthase. Circ Res 2000; 86: E36-E41.

10 Stoes E, Kastelein J, Cosentino F, Erkelens W, Wever R, Koomans H, Lüscher T, Rabelink T. Tetrahydrobiopterin restores endothelial function in hyper- cholesterolemia. J Clin Invest 1997; 99: 41-46.

11 Setoguchi S, Hirooka Y, Eshima K, Shimokawa H, Takeshita A. Tetrahydrobiopterin improves impaired endothelium-dependent forearm vasodilation in patients with heart failure. J Cardiovasc Pharmacol 2002; 39: 363-368.

12 Higashi Y, Sasaki S, Nakagawa K, Kimura M, Noma K, Hara K, Jitsuiki D, Goto C, Oshima T, Chayama K, Yoshizumi M. Tetrahydrobiopterin improves age-related impairment of endothelium- dependent vasodilation through increase in nitric oxide production and decrease in reactive oxygen species. Atherosclerosis 2006; 186: 390-395.

13 Inscho EW. P2 receptors in regulation of renal microvascular function. Am J Physiol Renal Physiol 2001; 280: F927-F944.

14 Heller R, Unbehaun A, Schellenberg B, Mayer B, Werner-Felmayer G, Werner ER. L-ascorbic acid potentiates endothelial nitric oxide synthesis via a chemical stabilization of tetrahydrobiopterin. J Biol Chem 2001; 276: 40-47.

15 Heitzer T, Schlinzig T, Krohn K, Meinertz T, Münzel T. Endothelial dysfunction, oxidative stress, and risk of cardiovascular events in patients with coronary artery disease. Circulation 2001; 104: 2673-2678.

16 Paolini M, Sapone A, Canistro D, Chieco P, Valgimigli L. Antioxidant vitamins for preservation of cardiovascular diseases. Lancet 2003; 362: 920. 